\title{
Comparative effectiveness of 5-hydroxytryptamine 3 receptor antagonists in irritable bowel syndrome: a network meta-analysis of randomized controlled studies
}

\author{
Theodore Rokkas ${ }^{\mathrm{a}, \mathrm{b}}$, Konstantinos Ekmektzoglou ${ }^{\mathrm{b}}$, Yaron Niv
}

Henry Dunant Hospital, Athens, Greece; European University of Cyprus, Nicosia, Cyprus; Tel Aviv University, Israel

Abstract

\begin{abstract}
Background There is evidence demonstrating the beneficial effects of 5-hydroxytryptamine 3 receptor antagonists $\left(5-\mathrm{HT}_{3}\right)$ for the treatment of non-constipated irritable bowel syndrome (NCIBS). We aimed to determine the comparative effectiveness of 5- $\mathrm{HT}_{3}$ antagonists in NC-IBS, as evidenced by the results of a network meta-analysis (NWM) of published relevant randomized controlled trials (RCTs).
\end{abstract}

Methods We searched the PubMed/Medline, EMBASE, and Cochrane Library databases for relevant RCTs through September 2020 and data from each selected RCT were extracted. A Bayesian NWM was then performed to investigate the efficacy of $5-\mathrm{HT}_{3}$ antagonists and to explore the effectiveness rank order in treating NC-IBS patients.

Results Twenty-one eligible RCTs were identified and entered into this NWM. They included a total of 10,421 NC-IBS patients, randomized to alosetron, cilansetron, ondansetron, ramosetron, placebo, and mebeverine. The cumulative ranking probability for each intervention at the end of treatment period, was evaluated by means of surfaces under cumulative ranking (SUCRA) values. These results showed that alosetron had the best performance for global symptom improvement (SUCRA 0.82), cilansetron showed the best performance (SUCRA 0.90) for abdominal pain/ discomfort improvement, while ondansetron (SUCRA 0.98) was by far the best choice concerning bowel habits/consistency improvement. The control regimens (mebeverine and placebo) represented the least efficacious interventions.

Conclusions This NWM showed that $5-\mathrm{HT}_{3}$ receptor antagonists performed better in comparison to control drugs. Consequently, this class of drugs may play an important role in improving the debilitating symptoms in NC-IBS patients, in particular those with diarrhea.

Keywords Irritable bowel syndrome, 5-hydroxytryptamine 3 receptor antagonists, alosetron, cilansetron, network meta-analysis

Ann Gastroenterol 2021; 34 (1): 1-13

\section{Introduction}

Irritable bowel syndrome (IBS) is characterized by chronic intermittent abdominal discomfort with concomitant diarrhea and/or constipation in patients who have no imaging, biochemical, or morphological abnormalities of

${ }^{\mathrm{a}}$ Gastroenterology Clinic, Henry Dunant Hospital, Athens, Greece (Theodore Rokkas); ${ }^{b}$ Medical School, European University of Cyprus, Nicosia, Cyprus (Theodore Rokkas, Konstantinos Ekmektzoglou); 'Tel Aviv University, Ministry of Health, Israel (Yaron Niv)

Conflict of Interest: None

Correspondence to: Theodore Rokkas, Gastroenterology Clinic, Henry Dunant Hospital, Athens 11526, Greece, e-mail: sakkor@otenet.gr

Received 18 November 2020; accepted 18 February 2021; published online 2 April 2021

DOI: https://doi.org/10.20524/aog.2021.0619 the gastrointestinal tract [1]. Patients with IBS suffer from frequent relapses that impair their quality of life. According to the results of the most recent epidemiological study using the Rome IV criteria, the global prevalence of IBS has been estimated to be $4.1 \%$ [2]. It is most common in women and age groups under 50 years old $[3,4]$. Concerning pathophysiology, studies have related IBS to altered intestinal microbiota, visceral hypersensitivity, irregular gastrointestinal motility, defects in the brain-gut axis and psychological factors [5-11]. Grossly, from the clinical point of view, IBS is classified into 3 main subtypes: i.e., IBS with diarrhea as the predominant manifestation (IBS-D), IBS where constipation prevails, and IBS with mixed bowel habits (IBS-M).

5-Hydroxytryptamine (5-HT) is mainly localized in the intestinal enterochromaffin cells and also in the brain $[9,10]$. There are seven subtypes of 5-HT receptors in the brain and gut. In the gut, $5-\mathrm{HT}_{3}$ receptors are situated mainly on intestinal plexuses, sensory nerves, sympathetic and parasympathetic nerves, where they stimulate neurotransmitter release [11].5- $\mathrm{HT}_{3}$ 
receptor antagonists (i.e., alosetron, cilansetron, ondansetron, and ramosetron) have been included in the therapeutic armamentarium for IBS patients, as they have been shown to benefit non-constipated IBS patients (NC-IBS). Consequently, their effectiveness has been assessed in randomized control clinical trials (RCTs) [12-32], in comparison to placebo [12,13,1527,29-32] and also a known anti-spasmodic agent (mebeverine) $[14,28]$. Some of these RCTs were included in 2 pairwise metaanalyses in the past $[33,34]$, whereas $25-\mathrm{HT}_{3}$ receptor antagonists (alosetron and ramosetron) were included in a systematic review and meta-analysis, which studied the efficacy of various pharmacological therapies in patients with IBS-D or IBS-M stool pattern [35]. However, the comparative effectiveness of all 5- $\mathrm{HT}_{3}$ receptor antagonists in patients with NC-IBS is unknown.

Network meta-analysis (NWM) has been used as an evidence synthesis tool for comparing RCTs with multiple treatments [36-38]. NWM incorporates both direct and indirect evidence in a collection of RCTs, thus providing information concerning the relative effects of 3 or more therapeutic interventions competing for a similar result. Since no NWM exists concerning the comparative effectiveness of all $5-\mathrm{HT}_{3}$ receptor antagonists evaluated in relevant RCTs, such a study is warranted. Our aim therefore was to perform an NWM in order to obtain more accurate and comprehensive results concerning the comparative efficacy of $5-\mathrm{HT}_{3}$ antagonists in the treatment of NC-IBS. It is expected that such comparisons will enable ranking of treatments and will help in clinical decision making.

\section{Materials and methods}

\section{Identification of studies and data extraction}

To identify studies and extract data in this NWM we followed the steps (i.e., identification, screening, eligibility, inclusion) described in our previous publications [39]. Thus, the PubMed/MEDLINE and Embase databases were searched through September 2020 to identify human studies written in English using the following search text and/or Medical Topic Heading (MeSH) terms: ("irritable bowel syndrome" $[\mathrm{MeSH}$ Terms] OR ("irritable"[All Fields] AND "bowel"[All Fields] AND "syndrome"[All Fields]) OR "irritable bowel syndrome"[All Fields]) AND ((("serotonin”[MeSH Terms] OR "serotonin"[All Fields]) AND type[All Fields] AND 3[All Fields] AND "receptor"[All Fields] AND ("antagonists and inhibitors"[Subheading] OR ("antagonists"[All Fields] AND "inhibitors"[All Fields]) OR "antagonists and inhibitors"[All Fields] OR “antagonists”[All Fields])) OR (5ht3[All Fields] AND ("antagonists and inhibitors"[Subheading] OR ("antagonists"[All Fields] AND “inhibitors"[All Fields]) OR "antagonists and inhibitors"[All Fields] OR "antagonists"[All Fields]. In addition, a manual search of all review articles, published editorials and retrieved original studies, was made. Two authors (TR and KE) independently extracted data from each study. Any disagreement was settled by further discussion with the third author (YN) until consensus was reached. This NWM was performed according to the PRISMA extension statement for interventions [40], while the quality of treatment effect estimates was rated using the GRADE (i.e., Grading of
Recommendations Assessment, Development and Evaluation) working group modality [41]. In addition, we appraised the confidence in estimates derived from NWM, as described in our previous publication [42]. In this process the construction of a matrix depicting the contribution of direct evidence to NWM results, together with a bar graph depicting the risk of bias for each network estimate and for the entire network, help in assessing the quality of evidence in the NWM.

\section{Selection criteria}

We defined the inclusion and exclusion criteria before starting the study investigation. Thus, appropriate studies were included in the meta-analysis provided that the following criteria were met: a) published as complete articles or abstracts with data that can be extracted; b) written in English; and c) were RCTs comparing $5-\mathrm{HT}_{3}$ antagonists in NC-IBS. Studies not meeting the above criteria were excluded. In this NWM 3 efficacy endpoints were taken into account: i.e., global improvement in NC-IBS symptoms; improvement in abdominal pain/discomfort; and improvement in bowel habits/consistency. In addition, one safety endpoint was evaluated, i.e., the constipation rate.

\section{Statistical analysis}

The $\mathrm{k}$ coefficient was used for the evaluation selection process by the reviewers. For pair-based meta-analyses and heterogeneity estimation we followed the methodology described previously [39]. In addition to heterogeneity, we assessed inconsistency, as this is critical when conducting an NWM [36-38]. We constructed comparison-adjusted funnel plots and checked their symmetry to assess whether smallscale trials influence the efficacy results. SUCRA (surfaces under cumulative ranking) values were used in intervention network charts to examine the cumulative ranking probability for each intervention in relation to the efficacy achieved by this intervention compared to an ideal intervention showing the best efficacy without doubt, i.e., SUCRA $=1$ or $100 \%$ when expressed as a percentage [36-38]. Data were processed using software suitable for Bayesian network meta-analysis, namely Stata 13.2 (StataCorp, College Station, TX) [36,37] and NetMetaX [38]. A value of $\mathrm{P}<0.05$ was the criterion of significance for all measurements except for heterogeneity, where the corresponding value was 0.1 to compensate for possible low power of the heterogeneity test [39].

\section{Results \\ Characteristics of studies}

The process of study selection is shown in Fig. 1. Thus, of 1308 titles yielded by the initial search, 21 RCTs [12-32] were finally eligible for meta-analysis. Reviewers' agreement concerning the selection of studies was high: $\mathrm{k}=0.94 ; 95 \%$ confidence interval [CI] 0.92-1). The characteristics of the 21 involved RCTs are shown in Table 1 . They were 18 full papers $[12-18,20,21,23,25-32]$ and 3 abstracts $[19,22,24]$. These 
studies included a total of 10,421 NC-IBS patients, of whom 9592 were IBS-D patients. These patients were randomized to placebo $(n=4,158)$, alosetron $(n=3,045)$, cilansetron $(n=1,116)$, ondansetron $(\mathrm{n}=147)$, ramosetron $(\mathrm{n}=1,483)$, and mebeverine $(\mathrm{n}=472)$ (Table 2). The 18 studies published as full papers [12$18,20,21,23,25-32]$ met all quality criteria. For the studies published as abstracts [19,22,24], a partial assessment of quality parameters was possible.

\section{NWM}

Of the 3 efficacy endpoints detailed above, 12 studies [14,18,19,21,22,24-26,27,29,31,32] reported on global improvement, 17 studies [12-17,19,20,22-29,31] reported on improvement in abdominal pain/discomfort, and 10 studies [12,15,20,25-31] reported on improvement in bowel habits/consistency. In all included RCTs the intention to treat analysis was taken into account.

\section{Network maps}

The network maps of all therapeutic interventions (alosetron, cilansetron, ramosetron, ondansetron, mebeverine, placebo) in relation to the 3 efficacy endpoints are depicted in
Fig. 2 (A,B,C). In these maps the node size reflects the number of patients allocated to each treatment, whereas the edge thickness is in proportion to the precision, i.e., the inverse of the variance of each direct comparison [36].

\section{Network plots}

The comparative effectiveness, expressed as odds ratio (OR), 95\% credible interval (CrI) of different therapeutic interventions, for the 3 efficacy endpoints is depicted in the respective forest plots of Fig. 3 (A,B,C). For the first efficacy endpoint, i.e., global symptom improvement (Fig. 3A), alosetron (OR 2.54, 95\% Crl 1.48-4.28) and cilansetron (OR 2.45, 95\% Crl 1.44-4.22) were significantly superior to placebo, whereas the respective value for ramosetron was (OR 1.44, 95\% Crl 0.95-2.24). For the second efficacy endpoint, i.e., improvement in abdominal pain/discomfort (Fig. 3B), cilansetron (OR 2.44, 95\% Crl 1.54-3.90) and alosetron (OR 1.60, 95\% Crl 1.18-2.14) were significantly superior to placebo, whereas the respective values for ondansetron and ramosetron did not reach significant levels (OR 1.98, 95\%Crl 0.57-6.56 and OR 1.34, 95\%Crl 0.91-1.98, respectively). For the third efficacy endpoint, i.e., improvement in bowel habits/ consistency (Fig. 3C), ondansetron (OR 5.50, 95\% Crl 2.90-

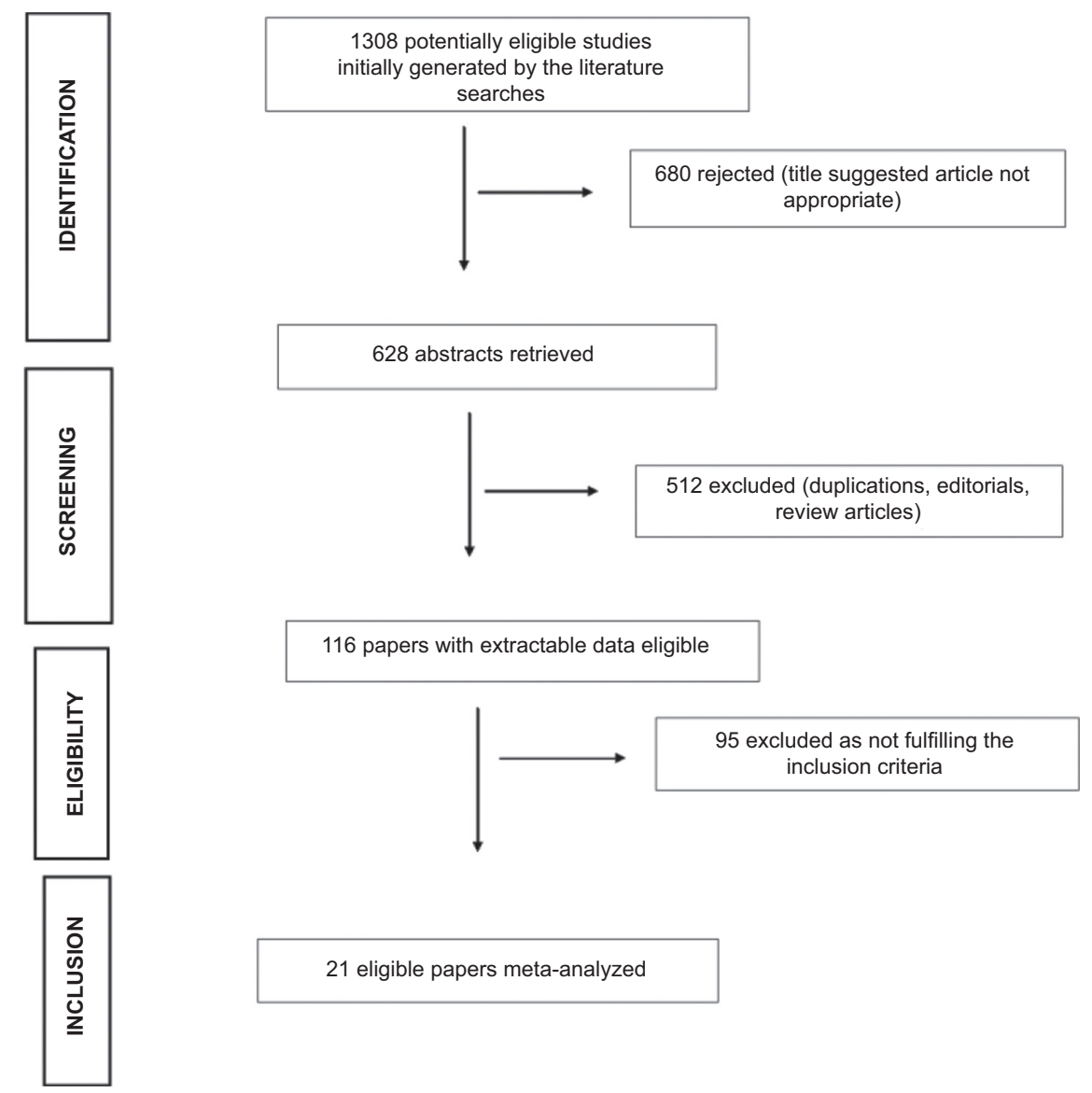

Figure 1 Flow chart of studies included in the network meta-analysis 


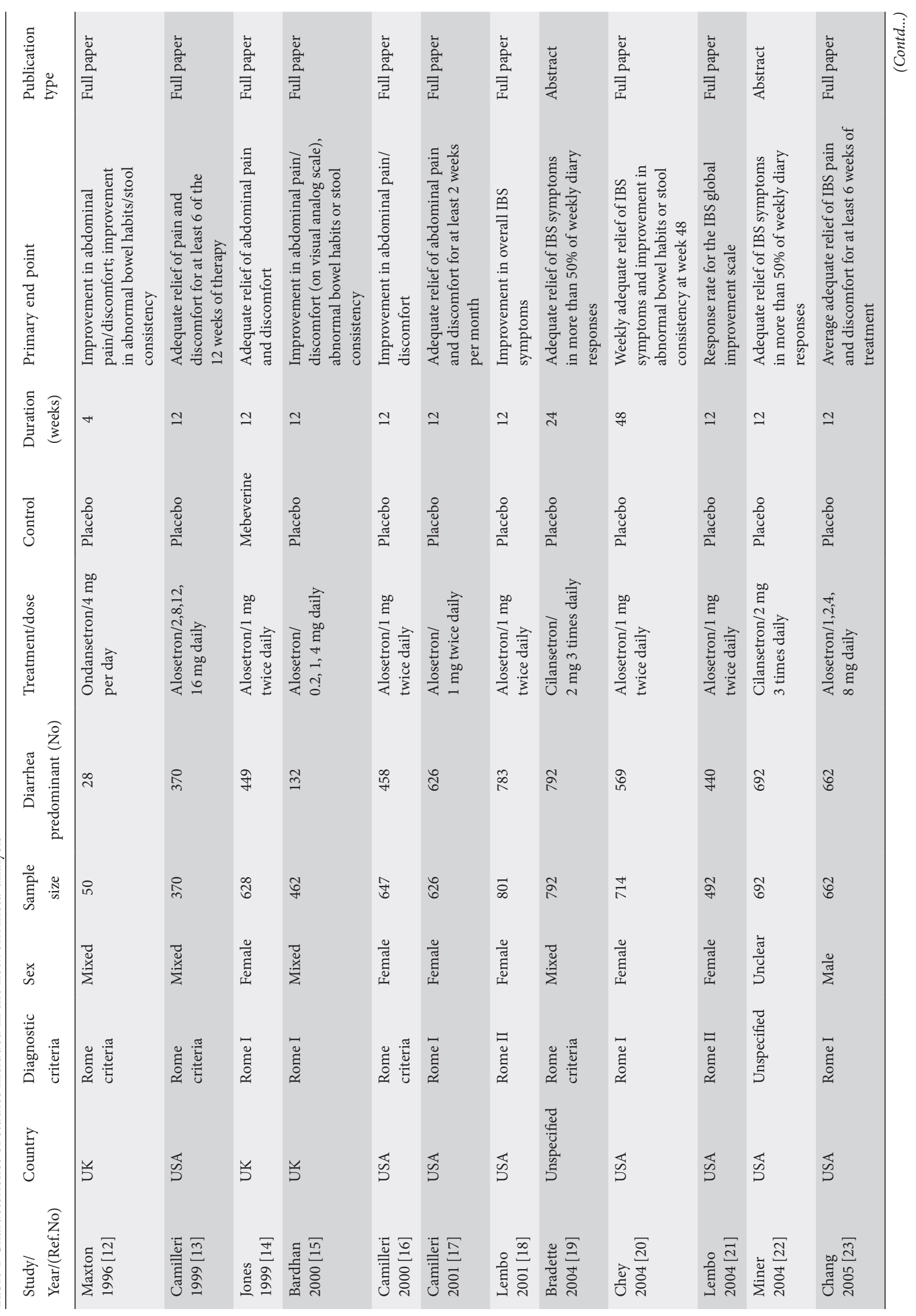




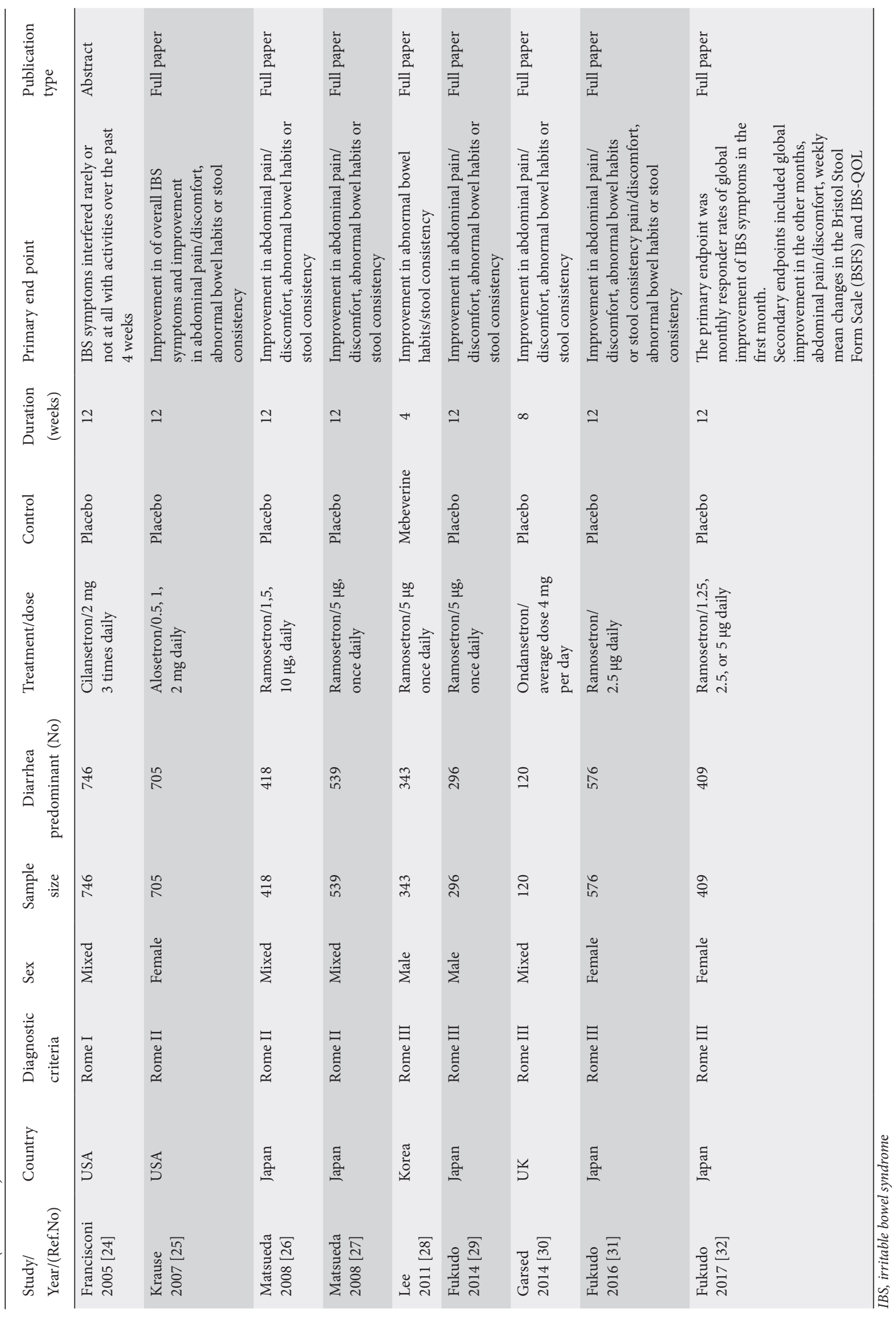


11.25) was by far superior to placebo, followed by alosetron (OR 2.57, 95\% Crl 1.83-3.77), and ramosetron (OR 2.12, 95\% Crl 1.53-2.95). In these network forest plots there was no significant heterogeneity; moreover, the evaluation of inconsistency yielded insignificant overall results, meaning that the comparative effect sizes obtained by direct and indirect comparisons were consistent. The relevant funnel plot (Fig. 4A) appeared symmetric, reflecting the lack of publication bias. The quality matrix of Fig. 4B depicts the summary of the risk of bias for each included study. The contribution of each comparison to the network is demonstrated in the constructed contribution plot (Fig. 4C) depicting the effects from all studies in the network. The bar graph of Fig. 4D illustrates the bias risk for

Table 2 Network characteristics and therapeutic interventions included in the network meta-analysis

\begin{tabular}{lc}
\hline Network Characteristics & No. \\
\hline Number of interventions & 6 \\
Number of studies & 21 \\
\hline Total number of patients in network & 10,421 \\
Total number of events in network & 4702 \\
\hline Total possible pairwise comparisons & 15 \\
Total number of pairwise comparisons with direct data & 6 \\
\hline Number of two-arm studies & 21 \\
\hline Number of multi-arm studies & 0 \\
\hline Number of studies with no zero events & 21 \\
Number of studies with at least one zero event & 0 \\
\hline Intervention & Studies \\
\hline Placebo & 19 \\
\hline Ramosetron & 6 \\
\hline Mebeverine & 2 \\
\hline Alosetron & 10 \\
\hline Cilansetron & 3 \\
\hline Ondansetron & 2 \\
\hline
\end{tabular}

each network assessment, showing the amount of information coming from studies with high, unclear and low risk of bias.

\section{League matrixes, rankograms and SUCRA values}

The comparative efficacies of the 6 therapeutic modalities (alosetron, cilansetron, ondansetron, ramosetron, mebeverine, and placebo) are shown in the league matrixes of Fig. 5 (A,B,C). These matrixes show that for global symptom improvement, alosetron performed better in comparison to cilansetron, mebeverine, ramosetron and placebo (Fig. 5A); for improvement in abdominal pain/discomfort, cilansetron performed better in comparison to alosetron, mebeverine, ramosetron and placebo (Fig. 5B); and finally, for improvement in bowel habits/consistency, ondansetron was by far superior in comparison to alosetron, ramosetron, mebeverine and placebo (Fig. $5 \mathrm{C}$ ). The respective rankograms are shown in Fig. 6 (A,B,C) in close relationship with the SUCRA values shown in Table 3. Thus, judging by the SUCRA values, these results showed that for global symptom improvement alosetron showed the best performance (SUCRA 0.82); for abdominal pain/discomfort cilansetron showed the best performance (SUCRA 0.90); and for improvement in bowel habits/consistency, ondansetron (SUCRA 0.98) was by far the best choice in comparison to other $5-\mathrm{HT}_{3}$ antagonists tested (i.e., alosetron SUCRA 0.64, ramosetron SUCRA 0.45) and also in comparison to mebeverine (SUCRA 0.40) and placebo (SUCRA 0.01 ), which represented the least efficacious regimens.

\section{Network plots for constipation}

The development of constipation is one of the main concerns when administrating 5- $\mathrm{HT}_{3}$ antagonists. The constipation report in the RCTs of this NWM is depicted in Supplementary Fig. $1(\mathrm{~A}, \mathrm{~B}, \mathrm{C})$, which show the network forest plot, the relevant league matrix and the rankogram, respectively. Participants in the intervention groups were more likely to develop constipation than those in the mebeverine or placebo groups. Thus, the ORs $(95 \% \mathrm{CI})$ for interventions that reported constipation, i.e., alosetron, cilansetron, ramosetron, were 5.28 (4.42-7.44), 3.38 (2.31-4.92), and 2.94 (1.91-4.49),

Table 3 SUCRA (surface under the cumulative ranking) values concerning the 6 therapeutic interventions for the 3 efficacy endpoints, i.e., global symptom improvement; improvement in abdominal pain/discomfort; and improvement in bowel habits/consistency

Efficacy endpoints

Global symptom improvement

\begin{tabular}{|c|c|c|c|c|c|}
\hline Treatment & SUCRA values & Treatment & SUCRA values & Treatment & SUCRA values \\
\hline Alosetron & 0.82 & Alosetron & 0.60 & Alosetron & 0.64 \\
\hline Cilansetron & 0.80 & Cilansetron & 0.90 & Cilansetron & - \\
\hline Ondansetron & - & Ondansetron & 0.66 & Ondansetron & 0.98 \\
\hline Ramosetron & 0.38 & Ramosetron & 0.42 & Ramosetron & 0.45 \\
\hline Mebeverine & 0.43 & Mebeverine & 0.31 & Mebeverine & 0.40 \\
\hline Placebo & 0.06 & Placebo & 0.10 & Placebo & 0.01 \\
\hline
\end{tabular}




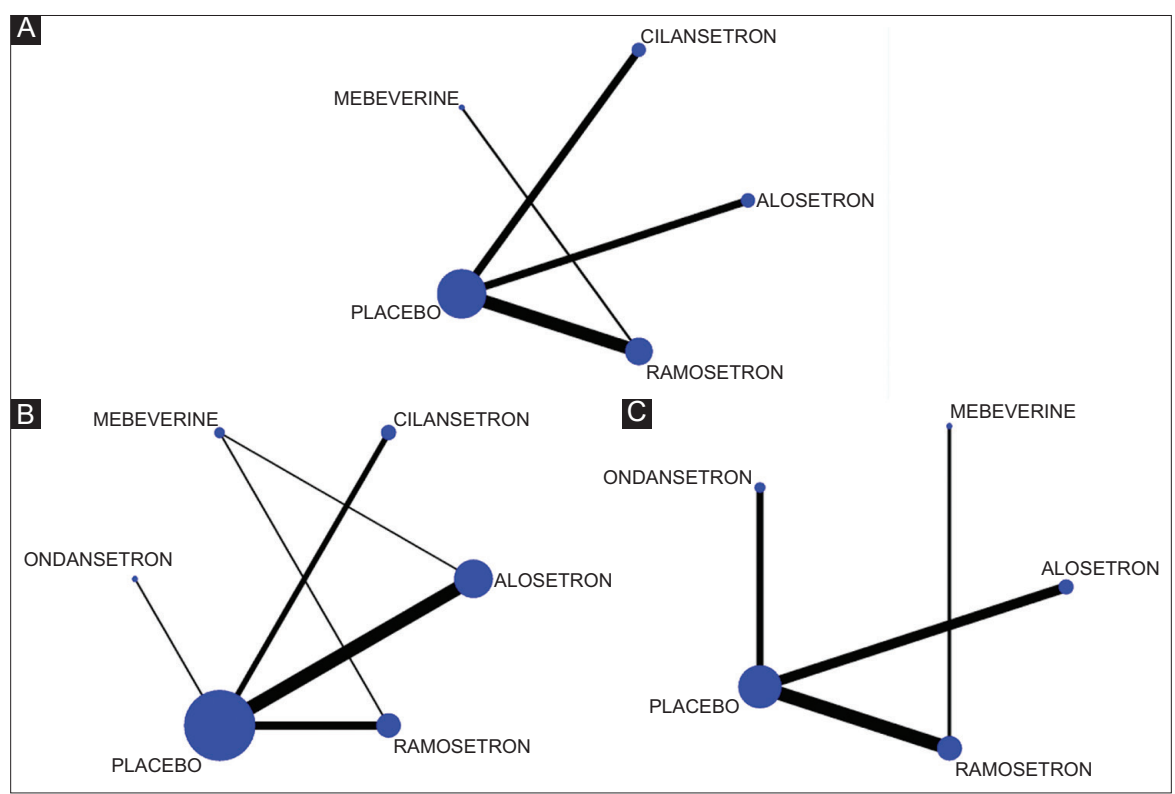

Figure 2 Network maps of the therapeutic interventions included in the randomized controlled trials. (A) Network map related to global improvement in non-constipated irritable bowel syndrome symptoms. (B) Network map related to improvement in abdominal pain/discomfort. (C) Network map related improvement in bowel habits/consistency. Node size reflects the number of patients randomly assigned to each treatment. Edge thickness is in proportion to the precision, i.e., the inverse of the variance of each direct comparison

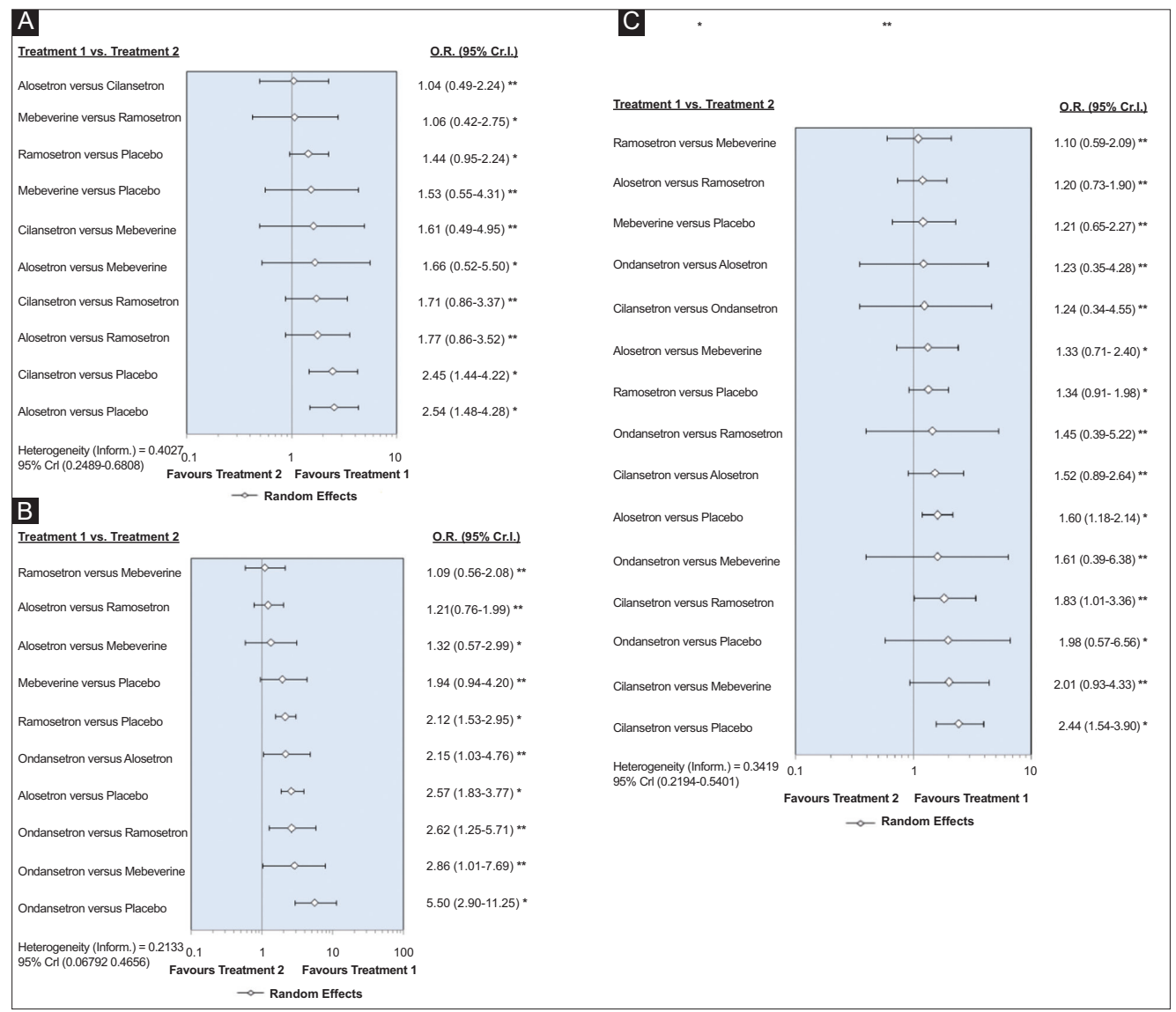

Figure 3 Forest plots illustrating all possible pairwise comparisons of therapeutic interventions included in the randomized controlled trials. (A) Global improvement in non-constipated irritable bowel syndrome symptoms. (B) Improvement in abdominal pain/discomfort. (C) Improvement in bowel habits/consistency. The horizontal lines represent the credible intervals

${ }^{*}=$ direct comparison, ${ }^{* *}=$ indirect comparison

OR, odds ratio; CrI, credible interval 


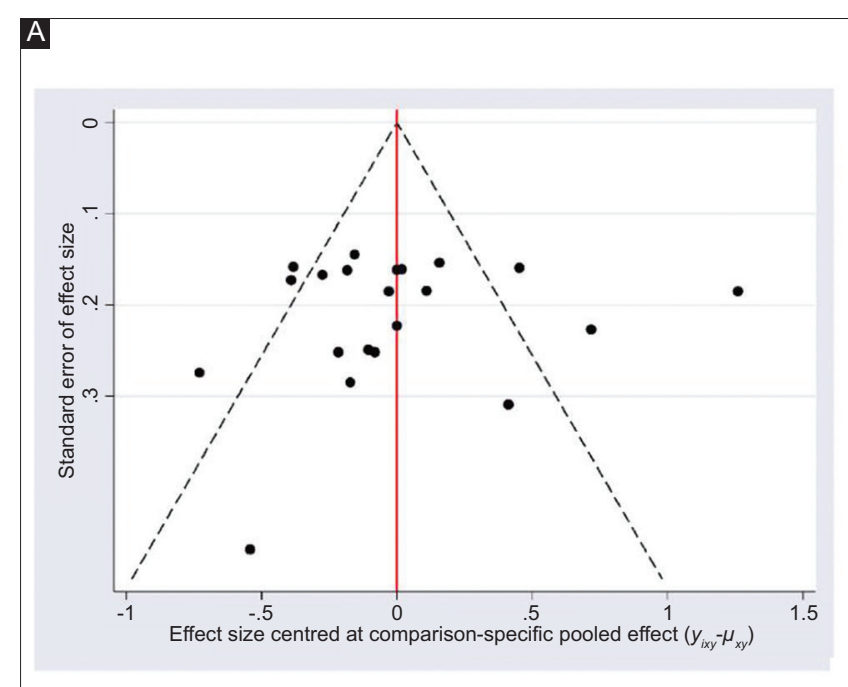

C

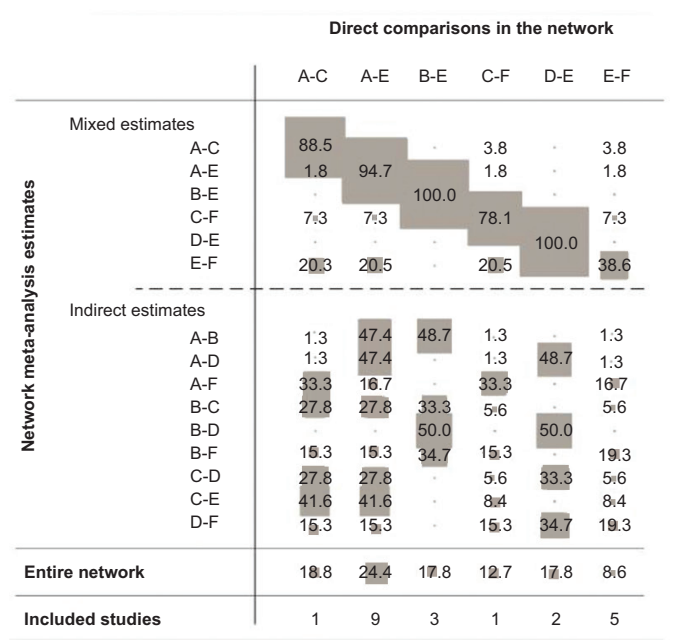

B

D
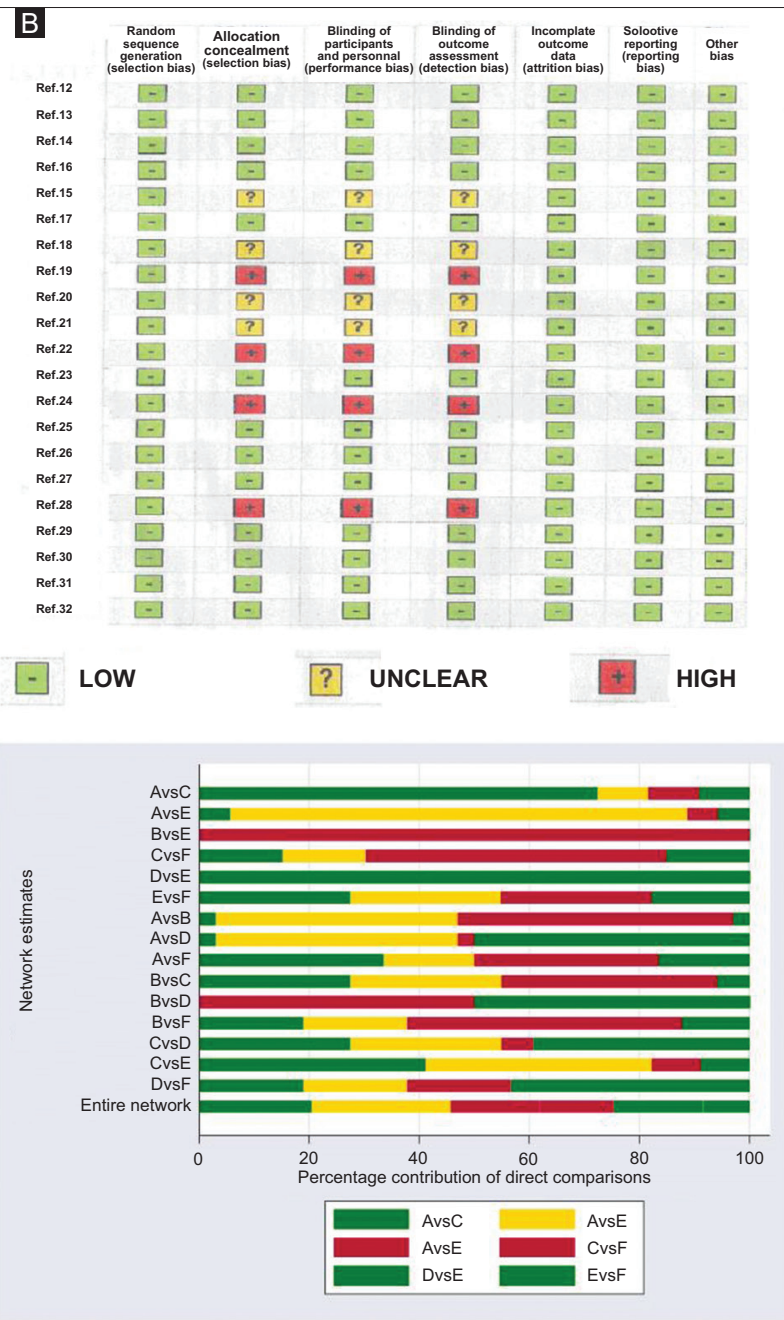

Figure 4 (A) Comparison-adjusted funnel plot. It appears symmetrical, meaning no publication bias. (B) Quality matrix depicting the summary of the risk of bias ( $\mathrm{RoB})$ for each included study. Studies are identified by the number in the reference list. (C) Contribution plot for the comparisons network. The numbers represent the percentage contribution of the column showing direct comparisons to the row defining network meta-analysis estimates. (D) Bar graph depicting the RoB for each network estimate

Treatment labels: A: alosetron; B: cilansetron; C: mebeverine; D: ondansetron; E: placebo; F: ramosetron

respectively, in comparison to placebo, and 11.43 (5.17-26.32), 6.90 (2.69-17.89), and 6.20 (2.28-14.80), respectively, in comparison to mebeverine.

\section{Discussion}

NC-IBS is a functional condition that affects the patient's quality of life, and IBS-D in particular could be debilitating due to urgency and episodic incontinence. Serotonin metabolism is involved in the pathophysiology of this condition and there are studies suggesting serotonin metabolism disturbance in these patients [9]. Interestingly, a recent study in IBS-D patients [43] found significantly increased mucosal concentrations of the 5-HT metabolite 5-hydroxyindole acetic acid (5-HIAA), together with an increased 5-HIAA/5-HT ratio, suggesting greater turnover of mucosal 5-HT in these patients. Among the various 5-HT receptors, $5-\mathrm{HT}_{3}$ receptors, localized on intestinal plexuses, sensory nerves, sympathetic and parasympathetic nerves, play a particular role in the pathophysiology of NC-IBS, stimulating the release of neurotransmitters. Consequently, research has focused on the therapeutic role of 5- $\mathrm{HT}_{3}$ receptor antagonists in NC-IBD, particularly in IBS-D. Therefore, $5-\mathrm{HT}_{3}$ receptor antagonists are a logical therapeutic option in IBS-D: this drug class currently includes alosetron, cilansetron, ramosetron and ondansetron. In this NWM we examined the comparative effectiveness of these $5-\mathrm{HT}_{3}$ receptor antagonists, taking into account all RCTs comparing them to control regimens (placebo and mebeverine). We studied 3 efficacy endpoints, i.e., global improvement in symptoms, improvement in abdominal pain/discomfort, and improvement in bowel habits/consistency. In addition, we studied constipation as a safety endpoint. 


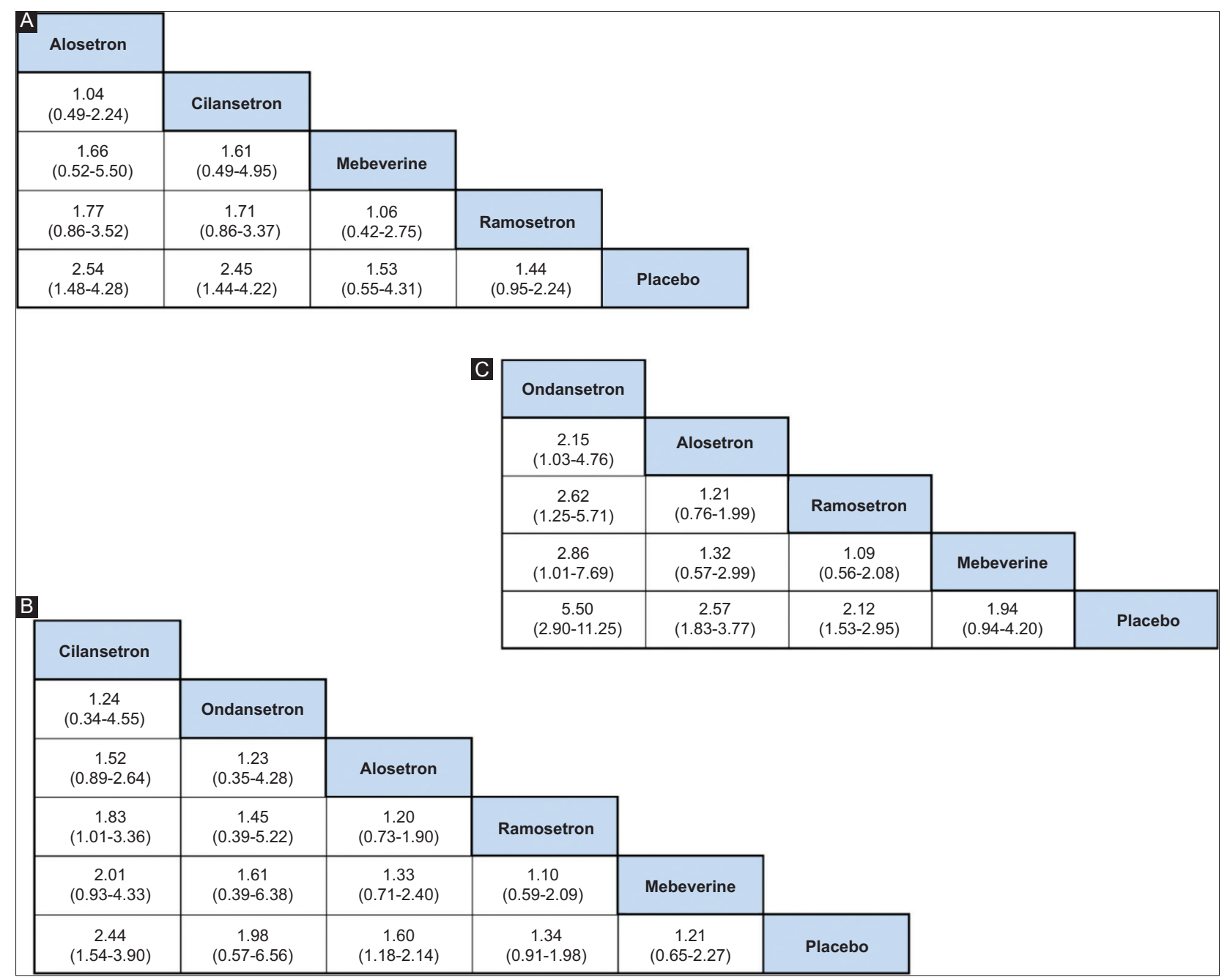

Figure 5 League matrixes showing the comparative efficacies of the therapeutic interventions included in the randomized controlled trials. (A) Global improvement in non-constipated irritable bowel syndrome symptoms. (B) Improvement in abdominal pain/discomfort. (C) Improvement in bowel habits/consistency

For RCTs with global improvement in symptoms as the main efficacy endpoint, the results showed that alosetron had the best efficacy in comparison to control drugs and to the other $5-\mathrm{HT}_{3}$ receptor antagonists studied. On the other hand, in these trials alosetron had the worse safety performance, as patients reported high constipation rates in comparison to those taking the other drugs examined. However, in all studies, constipation was considered mild to moderate in severity and in most cases resolved rapidly after the medication was stopped. Adverse events have been a main concern related to alosetron use: in fact, this drug was withdrawn in the USA because of adverse events, including ischemic colitis and severe constipation. It was reintroduced for female patients with severe IBS-D, and a 9-year evaluation concerning alosetron post-marketing safety reported 29 cases of probable ischemic colitis, i.e., an incidence of 1 case per 1000 patient-years [44]. This rate was similar to the background rate of ischemic colitis in female IBS patients, which ranged from 0.4-1.79 cases per 1000 patient-years [45]. The effectiveness of alosetron in male patients with NC-IBS is not clear, since only one of the RCTs included in this NWM recruited males only [23], whereas the other RCTs included exclusively or predominantly female patients. Cilansetron showed the best efficacy in RCTs when improvement in abdominal pain/discomfort was the main efficacy endpoint, and it appeared to be effective in both male and female patients. However, despite the good results concerning the improvement in abdominal pain/discomfort, this $5-\mathrm{HT}_{3}$ receptor antagonist carries 2 main weaknesses: all 3 relevant RCTs included in this NWM were abstracts and were never published as full papers, and in addition the drug never came to market. In this NWM ramosetron showed low performance and proved to be more effective than control drugs [OR 2.12 (95\% Crl 1.53-2.95)] only in improving abdominal pain/discomfort, with no serious related adverse event, specifically ischemic colitis. However, it must be noted that all relevant RCTs were conducted in Japan; thus, these findings might not be generalizable to patients with NC-IBS in the USA and Europe. Indeed, at present ramosetron is only licensed in Japan and some other Asian countries, at a dose of $2.5 \mu \mathrm{g}$ q.d. for females and $5 \mu \mathrm{g}$ q.d. for males. Therefore, NC-IBS patients in western countries will be unable to access this drug. 

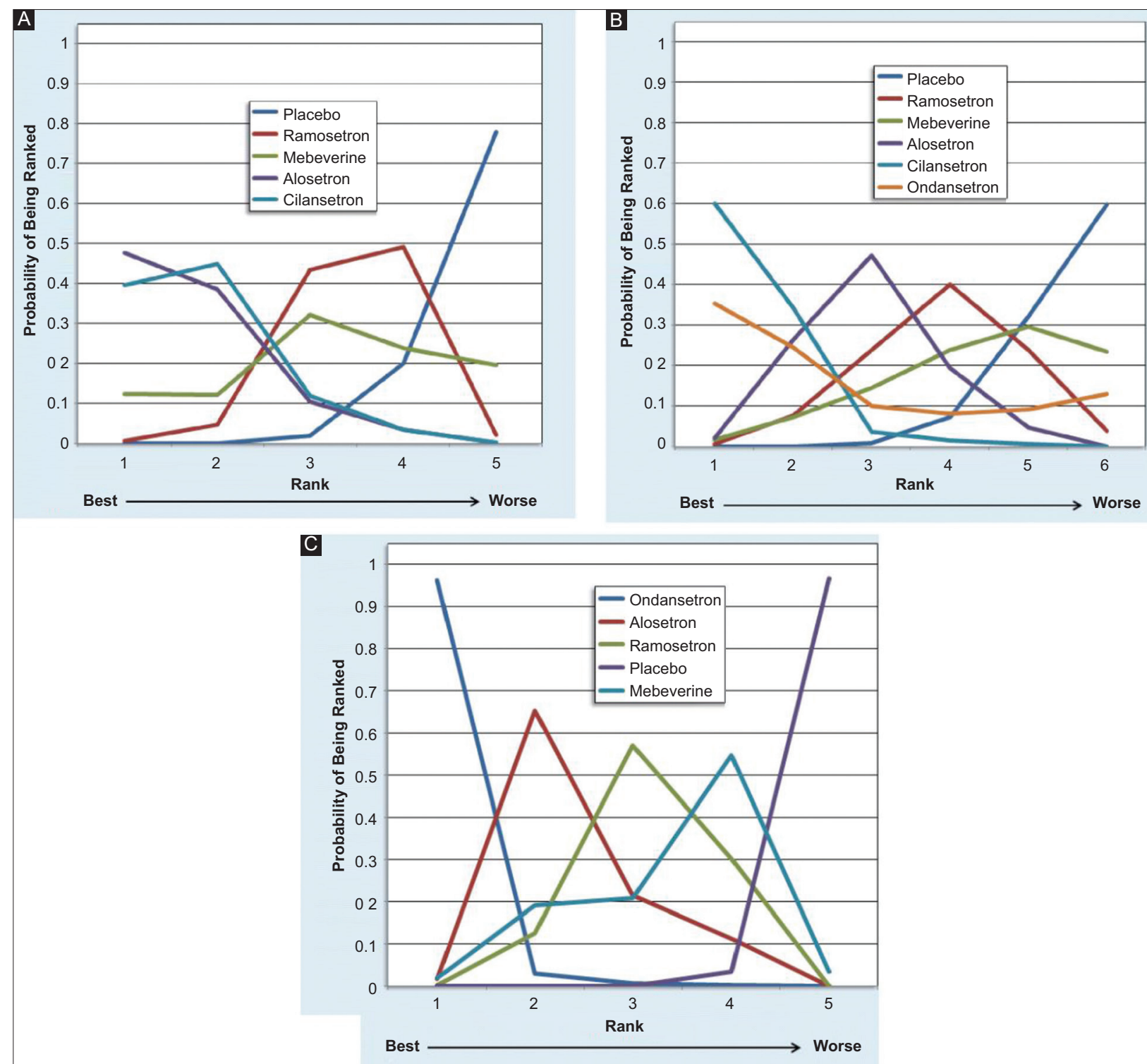

Figure 6 Rankogram networks for the therapeutic interventions included in the randomized controlled trials, showing the cumulative rank order for each intervention. (A) Global improvement in non-constipated irritable bowel syndrome symptoms. (B) Improvement in abdominal pain/ discomfort. (C) Improvement in bowel habits/consistency

Ondansetron, in comparison to other $5-\mathrm{HT}_{3}$ receptor antagonists, performed better when bowel habits/consistency were the main efficacy endpoint. This $5-\mathrm{HT}_{3}$ receptor antagonist, is currently licensed for use in both adults and children, mainly for the management of nausea and vomiting induced by chemotherapy and radiotherapy, and for postoperative nausea and vomiting. One point of considerable concern is that, in this NWM, all evaluations concerning ondansetron were based on only 2 RCTs that included a total of 170 patients. All these observations point to a need for large well-designed RCTs to better define the efficacy and optimal dose of ondansetron in NC-IBS patients. Along these lines, a large multicenter RCT (the TRITON trial) is in progress [46] and is expected to be completed by December 2021. Of considerable importance is the fact that in this trial several mechanistic assessments, such as gut transit, fecal tryptase and fecal bile acid concentrations, barostat and high resolution rectal manometry tests, will be included in an effort to better define ondansetron's mechanisms of action. As the authors of this study protocol claim, if ondansetron proved to be effective in this well-designed trial, then it could easily be widely adopted, since it is an inexpensive, safe and generic drug. Therefore, by providing an effective treatment, it could not only reduce patient symptoms, but also reduce healthcare costs associated with repeated referrals and unnecessary investigations.

As far as the potential mechanisms of action of $5-\mathrm{HT}_{3}$ receptor antagonists is concerned, there is evidence that the beneficial effect of this drug class in NC-IBS might be related to the prolongation of gastrointestinal transit time and improvement in rectal compliance $[47,48]$, as well as a reduction in visceral 


\section{Summary Box}

\section{What is already known:}

- There is evidence demonstrating the beneficial effects of 5-hydroxytryptamine receptor $\left(5-\mathrm{HT}_{3}\right)$ antagonists for the treatment of non-constipated irritable bowel syndrome (NC-IBS)

- The effectiveness of alosetron, cilansetron, ondansetron and ramosetron has been assessed in randomized control clinical trials (RCTs) in comparison to control regimens (placebo and mebeverine)

- Currently the comparative effectiveness of all $5-\mathrm{HT}_{3}$ receptor antagonists in patients with NCIBS is lacking

\section{What the new findings are:}

- This network meta-analysis showed that all $5-\mathrm{HT}_{3}$ receptor antagonists performed better in comparison to control regimens

- Alosetron showed the best performance for global symptom improvement, cilansetron showed the best performance for abdominal pain/discomfort, and ondansetron was by far the best choice for improvement in bowel habits/consistency, in comparison to other $5-\mathrm{HT}_{3}$ antagonists and also to control regimens (mebeverine and placebo), which represented the least efficacious regimens

- Consequently, this class of drugs may play an important role in improving the debilitating symptoms in NC-IBS patients, in particular those with diarrhea

sensitivity [49]. However, more studies including various mechanistic assessments, as discussed above, are necessary to better define the mode of action of this drug category.

The lack of significant inconsistency in this NWM strengthens our results. However, despite the messages that emerge concerning the effectiveness of $5-\mathrm{HT}_{3}$ receptor antagonists vs. control drugs (placebo and mebeverine), some limitations should be stressed. The main limitation is related to differences in the design and endpoints used in individual RCTs, as well as to the heterogeneity concerning the Rome criteria used in the individual RCTs, i.e., Rome I vs. Rome II vs. Rome III criteria. In future RCTs these concerns are expected to be addressed by utilizing the standardized FDA-recommended endpoints for patients with IBS-D. Another limitation refers to the fact that some RCTs included only a small number of patients, in particular for evaluations concerning ondansetron. However, a large, welldesigned multicenter RCT (the TRITON trial) [46] addresses the ondansetron issue and this trial is expected to be completed soon. A recent systematic review [35] addressed the efficacy of various pharmacological therapies (alosetron, ramosetron, rifaximin and eluxadoline) in patients who had IBS-D or IBS-M stool pattern and found that alosetron and ramosetron were the most effective.
As the authors stress, although the 5- $\mathrm{HT}_{3}$ receptor antagonists appeared to perform the best, many IBS patients will be unable to access these drugs. However, drug availability will no longer be a problem if the TRITON trial results fulfill the expectations concerning ondansetron.

In conclusion, the results of this NWM show that $5-\mathrm{HT}_{3}$ receptor antagonists play an important role in improving the debilitating symptoms related to NC-IBS, in particular IBS-D, given the associated urgency and episodic incontinence. These findings are expected to be taken into account when considering therapeutic options for IBS. However, since there are a number of questions still to be answered, well-designed RCTs are needed to confirm the efficacy, safety profile and optimal dose of 5- $\mathrm{HT}_{3}$ receptor antagonists in IBS patients.

\section{References}

1. Longstreth GF, Thompson WG, Chey WD, Houghton LA, Mearin F, Spiller RC. Functional bowel disorders. Gastroenterology 2006;130:1480-1491.

2. Sperber AD, Bangdiwala SI, Drossman DA, et al. Worldwide prevalence and burden of functional gastrointestinal disorders, results of Rome foundation global study. Gastroenterology 2021;160:99-114.e3.

3. Grundmann O, Yoon SL. Irritable bowel syndrome: epidemiology, diagnosis and treatment: an update for health-care practitioners. J Gastroenterol Hepatol 2010;25:691-699.

4. Krogsgaard LR, Engsbro AL, Bytzer P. The epidemiology of irritable bowel syndrome in Denmark. A population-based survey in adults $\leq 50$ years of age. Scand J Gastroenterol 2013;48:523-529.

5. Lee YJ, Park KS. Irritable bowel syndrome: emerging paradigm in pathophysiology. World J Gastroenterol 2014;20:2456-2469.

6. Barbara G, De Giorgio R, Stanghellini V, Cremon C, Salvioli B, Corinaldesi R. New pathophysiological mechanisms in irritable bowel syndrome. Aliment Pharmacol Ther 2004;20 Suppl 2:1-9.

7. Posserud I, Stotzer PO, Björnsson ES, Abrahamsson H, Simrén M. Small intestinal bacterial overgrowth in patients with irritable bowel syndrome. Gut 2007;56:802-808.

8. Lacy BE, Mearin F, Chang L, et al. Bowel disorders. Gastroenterology 2016;150:1393-1407.e5.

9. Atkinson W, Lockhart S, Whorwell PJ, Keevil B, Houghton LA. Altered 5-hydroxytryptamine signaling in patients with constipation- and diarrhea-predominant irritable bowel syndrome. Gastroenterology 2006;130:34-43.

10. Berger M, Gray JA, Roth BL. The expanded biology of serotonin. Annu Rev Med 2009;60:355-366.

11. Gershon MD, Wade PR, Kirchgessner AL, Tamir H. 5-HT receptor subtypes outside the central nervous system. Roles in the physiology of the gut. Neuropsychopharmacology 1990;3:385-395.

12. Maxton DG, Morris J, Whorwell PJ. Selective 5-hydroxytryptamine antagonism: a role in irritable bowel syndrome and functional dyspepsia? Aliment Pharmacol Ther 1996;10:595-599.

13. Camilleri M, Mayer EA, Drossman DA, et al. Improvement in pain and bowel function in female irritable bowel patients with alosetron, a 5-HT3 receptor antagonist. Aliment Pharmacol Ther 1999;13:1149-1159.

14. Jones RH, Holtmann G, Rodrigo L, et al. Alosetron relieves pain and improves bowel function compared with mebeverine in female nonconstipated irritable bowel syndrome patients. Aliment Pharmacol Ther 1999;13:1419-1427.

15. Bardhan KD, Bodemar G, Geldof $\mathrm{H}$, et al. A double-blind, randomized, placebo-controlled dose-ranging study to evaluate the efficacy of alosetron in the treatment of irritable bowel syndrome. 
Aliment Pharmacol Ther 2000;14:23-34.

16. Camilleri M, Northcutt AR, Kong S, Dukes GE, McSorley D, Mangel AW. Efficacy and safety of alosetron in women with irritable bowel syndrome: a randomised, placebo-controlled trial. Lancet 2000;355:1035-1040.

17. Camilleri M, Chey WY, Mayer EA, et al. A randomized controlled clinical trial of the serotonin type 3 receptor antagonist alosetron in women with diarrhea-predominant irritable bowel syndrome. Arch Intern Med 2001;161:1733-1740.

18. Lembo T, Wright RA, Bagby B, et al; Lotronex Investigator Team. Alosetron controls bowel urgency and provides global symptom improvement in women with diarrhea-predominant irritable bowel syndrome. Am J Gastroenterol 2001;96:2662-2670.

19. Bradette M, Moennikes H, Carter F, et al. Cilansetron in irritable bowel syndrome with diarrhea predominance (IBS-D): efficacy and safety in a 6-month global study. Gastroenterology 2004;126(Suppl 2):A42.

20. Chey WD, Chey WY, Heath AT, et al. Long-term safety and efficacy of alosetron in women with severe diarrhea-predominant irritable bowel syndrome. Am J Gastroenterol 2004;99:2195-2203.

21. Lembo AJ, Olden KW, Ameen VZ, Gordon SL, Heath AT, Carter EG. Effect of alosetron on bowel urgency and global symptoms in women with severe, diarrhea-predominant irritable bowel syndrome: analysis of two controlled trials. Clin Gastroenterol Hepatol 2004;2:675-682.

22. Miner P, Stanton D, Carter F, et al. Cilansetron in irritable bowel syndrome with diarrhea predominance (IBS-D): efficacy and safety in a 3 month US study. Am J Gastroenterol 2004;99:S277.

23. Chang L, Ameen VZ, Dukes GE, McSorley DJ, Carter EG, Mayer EA. A dose-ranging, phase II study of the efficacy and safety of alosetron in men with diarrhea-predominant IBS. Am J Gastroenterol 2005;100:115-123.

24. Francisconi CF, Drossman DA, Mayer EA, et al. Interruption of daily activities in cilansetron-treated patients with irritable bowel syndrome with diarrhea-predominance (IBS-D): results from a 16week, placebo-controlled, randomization trial. Gastroenterology 2006;130(Suppl 2):A600.

25. Krause R, Ameen V, Gordon SH, et al. A randomized, double-blind, placebo-controlled study to assess efficacy and safety of $0.5 \mathrm{mg}$ and $1 \mathrm{mg}$ alosetron in women with severe diarrhea-predominant IBS. Am J Gastroenterol 2007;102:1709-1719.

26. Matsueda K, Harasawa S, Hongo M, Hiwatashi N, Sasaki D. A phase II trial of the novel serotonin type 3 receptor antagonist ramosetron in Japanese male and female patients with diarrheapredominant irritable bowel syndrome. Digestion 2008;77:225-235.

27. Matsueda K, Harasawa S, Hongo M, Hiwatashi N, Sasaki D. A randomized, double-blind, placebo-controlled clinical trial of the effectiveness of the novel serotonin type 3 receptor antagonist ramosetron in both male and female Japanese patients with diarrhea-predominant irritable bowel syndrome. Scand $J$ Gastroenterol 2008;43:1202-1211.

28. Lee KJ, Kim NY, Kwon JK, et al. Efficacy of ramosetron in the treatment of male patients with irritable bowel syndrome with diarrhea: a multicenter, randomized clinical trial, compared with mebeverine. Neurogastroenterol Motil 2011;23:1098-1104.

29. Fukudo S, Ida M, Akiho H, Nakashima Y, Matsueda K. Effect of ramosetron on stool consistency in male patients with irritable bowel syndrome with diarrhea. Clin Gastroenterol Hepatol 2014;12:953-959.e4.

30. Garsed K, Chernova J, Hastings M, et al. A randomised trial of ondansetron for the treatment of irritable bowel syndrome with diarrhea. Gut 2014;63:1617-1625.

31. Fukudo S, Kinoshita Y, Okumura T, et al. Ramosetron reduces symptoms of irritable bowel syndrome with diarrhea and improves quality of life in women. Gastroenterology 2016;150:358-366.e8.
32. Fukudo S, Matsueda K, Haruma K, et al. Optimal dose of ramosetron in female patients with irritable bowel syndrome with diarrhea: A randomized, placebo-controlled phase II study. Neurogastroenterol Motil 2017;29:e13023.

33. Andresen V, Montori VM, Keller J, West CP, Layer P, Camilleri M. Effects of 5-hydroxytryptamine (serotonin) type 3 antagonists on symptom relief and constipation in nonconstipated irritable bowel syndrome: a systematic review and meta-analysis of randomized controlled trials. Clin Gastroenterol Hepatol 2008;6:545-555.

34. Zheng Y, Yu T, Tang Y, et al. Efficacy and safety of 5-hydroxytryptamine 3 receptor antagonists in irritable bowel syndrome: A systematic review and meta-analysis of randomized controlled trials. PLoS One 2017;12:e172846.

35. Black CJ, Burr NE, Camilleri M, et al. Efficacy of pharmacological therapies in patients with IBS with diarrhoea or mixed stool pattern: systematic review and network meta-analysis. Gut 2020;69:74-82.

36. Salanti G, Ades AE, Ioannidis JP. Graphical methods and numerical summaries for presenting results from multiple-treatment metaanalysis: an overview and tutorial. J Clin Epidemiol 2011;64:163-171.

37. Chaimani A, Higgins JP, Mavridis D, Spyridonos P, Salanti G. Graphical tools for network meta-analysis in STATA. PLoS One 2013;8:e76654.

38. Brown S, Hutton B, Clifford T, et al. A Microsoft-Excel-based tool for running and critically appraising network meta-analyses-an overview and application of NetMetaXL. Syst Rev 2014;3:110.

39. Rokkas T, Gisbert JP, Niv Y, O'Morain C. The association between Helicobacter pylori infection and inflammatory bowel disease based on meta-analysis. United European Gastroenterol J 2015;3:539-550.

40. Hutton B, Salanti G, Caldwell DM, et al. The PRISMA extension statement for reporting of systematic reviews incorporating network meta-analyses of health care interventions: checklist and explanations. Ann Intern Med 2015;162:777-784.

41. Puhan MA, Schünemann HJ, Murad MH, et al; GRADE Working Group. A GRADE Working Group approach for rating the quality of treatment effect estimates from network meta-analysis. BMJ 2014;349:g5630.

42. Rokkas T, Gisbert JP, Gasbarrini A, et al. A network meta-analysis of randomized controlled trials exploring the role of fecal microbiota transplantation in recurrent Clostridium difficile infection. United European Gastroenterol J 2019;7:1051-1063.

43. Gunn D, Garsed K, Lam C, et al. Abnormalities of mucosal serotonin metabolism and 5-HT3 receptor subunit 3C polymorphism in irritable bowel syndrome with diarrhea predict responsiveness to ondansetron. Aliment Pharmacol Ther 2019;50:538-546.

44. Tong K, Nicandro JP, Shringarpure R, Chuang E, Chang L. A 9-year evaluation of temporal trends in alosetron postmarketing safety under the risk management program. Therap Adv Gastroenterol 2013;6:344-357.

45. Cole JA, Cook SF, Sands BE, Ajene AN, Miller DP, Walker AM. Occurrence of colon ischemia in relation to irritable bowel syndrome. Am J Gastroenterol 2004;99:486-491.

46. Gunn D, Fried R, Lalani R, et al. Treatment of irritable bowel syndrome with diarrhoea using titrated ondansetron (TRITON): study protocol for a randomised controlled trial. Trials 2019;20:517.

47. Houghton LA, Foster JM, Whorwell PJ. Alosetron, a 5-HT3 receptor antagonist, delays colonic transit in patients with irritable bowel syndrome and healthy volunteers. Aliment Pharmacol Ther 2000; 14:775-782.

48. Min YW, Rhee PL. The clinical potential of ramosetron in the treatment of irritable bowel syndrome with diarrhea (IBS-D). Therap Adv Gastroenterol 2015;8:136-142.

49. Delvaux M, Louvel D, Mamet JP, Campos-Oriola R, Frexinos J. Effect of alosetron on responses to colonic distension in patients with irritable bowel syndrome. Aliment Pharmacol Ther 1998; 12:849-855. 


\section{Supplementary material}

A

$\underline{\text { Treatment } 1 \text { vs. Treatment } 2}$

Cilansetron versus Ramosetron

Alosetron versus Cilansetron

Alosetron versus Ramosetron

Placebo versus Mebeverine

Ramosetron versus Placebo

Cilansetron versus Placebo

Alosetron versus Placebo

Ramosetron versus Mebeverine

Cilansetron versus Mebeverire

Alosetron versus Mebeverine

Heterogeneity (inform.) $=0.17210 .1$ $95 \% \mathrm{Crl}(0.05298-0.3983)$

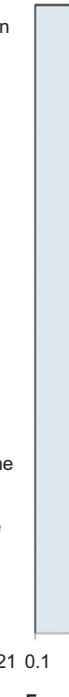

Favours Treatment 2 Favours Treatment 1
B

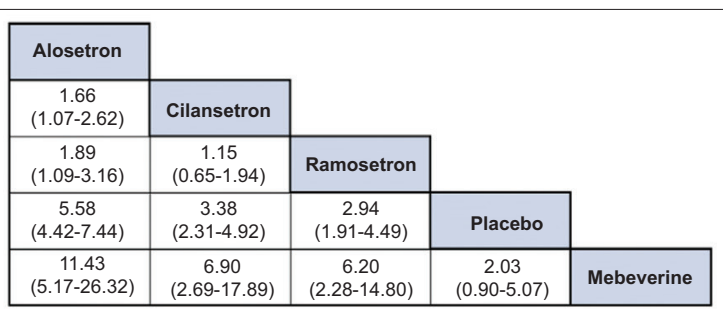

C

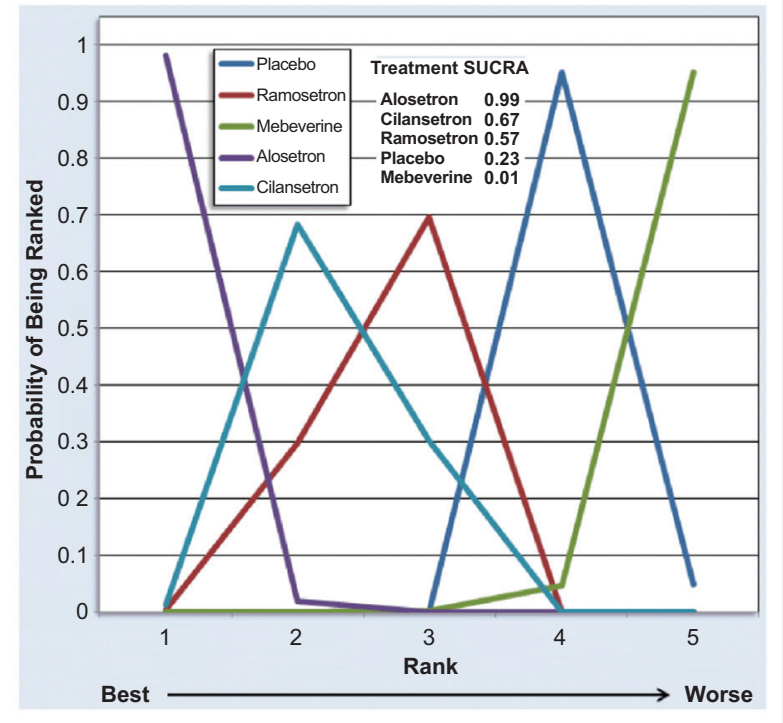

Supplementary Figure 1 Constipation report concerning the treatments included in the RCTs of the NWM. (A) Forest plot illustrating pairwise comparisons. The horizontal lines represent the credible intervals (CrI). (B) League matrix showing the comparative performance of the treatments. (C) Rankogram network for the therapeutic interventions included in the RCTs, showing the cumulative rank order for each intervention. SUCRA (surface under the cumulative ranking) values for the competing 5 therapeutic interventions

${ }^{*}=$ direct comparison, ${ }^{* *}=$ indirect comparison.

5-HT3, 5-hydroxytryptamine 3 receptor antagonist; NWM, network meta-analysis; IBS, irritable bowel syndrome; RCT, randomized controlled trial; OR, odds ratio 